\title{
Primary Upper Extremity Deep Venous Thrombosis and Comorbidity: A Population-Based Nationwide Study in Sweden
}

\author{
Authors: \\ Thomas Arnhjort*, Gerd Lärfars*, Johanna Adami**, Hans Järnbert Pettersson* \\ * Department of Clinical Science and Education, Södersjukhuset, Karolinska Institutet, \\ Stockholm, Sweden \\ **`Clinical Epidemiology Unit, Department of Medicine, Karolinska Institutet, Stockholm, \\ Sweden
}

\section{Corresponding Author:}

Thomas Arnhjort

$\mathrm{MD}, \mathrm{PhD}$, Consultant

Älgöbranten 2

13337 Saltsjöbaden

E-mail: thomas.arnhjort@telia.com

Phone: +46707362798

\section{Short title: Primary arm thrombosis - a register study}

\section{Compliance with Ethical Standards and funding:}

This study was funded by Skandinaviska Forskningsstifelsen för Åderbråck och Vensjukdomar (Scandinavian research Foundation for varicose veins and venous diseases)

Conflict of Interest: None of the authors declare any conflict of interest.

Ethical approval: All procedures performed in this study were in accordance with the ethical standards of the national research committee and with the 1964 Helsinki declaration and its later amendments or comparable ethical standards.

The article is a register study and informed consent is not mandatory according to the Regional Ethical Review Board in Stockholm, Sweden. 


\section{Abstract \\ Background}

There have been few studies on primary upper extremity deep vein thrombosis (UEDVT) and the comorbidity is not very well known, in contrast to secondary UEDVT.

\section{Methods}

We conducted a nationwide, population-based case control study in Sweden using data from the Swedish National Patient Register (NPR).

We examined comorbidity in 25 cases with a confirmed UEDVT with a control group (appendicitis) during the years 2001-2011 in patients over 18 years old. We defined comorbidity as the number of unique ICD codes (International Statistical Classification of Diseases and Related Health Problems) and the total number of healthcare contacts.

\section{Results}

The number of unique ICD codes was similar between cases and controls, although they differed regarding specific diagnoses. Patients with a UEDVT were more likely to later have a diagnosis related to blood-forming organs, the endocrine system, the nervous system, the circulatory system, and to the skin and musculoskeletal systems, compared with the controls.

Diagnoses for symptoms from the brachial plexus and diagnoses referring to pain and discomfort of the upper extremity were more frequent in the cases. The major difference occurred in diagnoses involving the circulatory system, which were more frequent in the cases. The most important cases and controls were similar regarding diagnoses of malignancy. The cases had a significantly higher number of healthcare contacts.

\section{Conclusion}

The type of comorbidity and the number of healthcare contacts differed between patients with a diagnosis of UEDVT, compared with those with appendicitis. There was no difference regarding malignancies, which implies that primary UEDVT is a benign disorder.

Key words: Primary upper extremity deep vein thrombosis, Register study, Comorbidity 


\section{Introduction}

Upper extremity deep vein thrombosis (UEDVT) is defined as an acute and painful swelling of the arm resulting from thrombosis of the veins of the upper extremity. The proximal veins of the shoulder and arm are the most common location for UEDVT. Both the subclavian and axillary veins [1-3] and the brachial vein may be involved [4]. The frequency of UEDVT varies in different studies, and the proportion is $1-10 \%$ of all DVTs [5-8].

UEDVT can be classified into two groups, primary and secondary. There are very few studies on primary UEDVT and its long-term consequences. The comorbidity in patients with primary UEDVT is not very well known, in contrast to secondary UEDVT, where the comorbidity has been well described.

The aim of this case control study was to compare the comorbidity in patients with primary UEDVT treated with low molecular weight heparin (LMWH) and vitamin K antagonists (VKAs) with that of a control group.

\section{Background}

Primary UEDVT includes idiopathic and effort-related UEDVT. Some studies suggest that up to one third of all UEDVTs could be classified as primary cases, whereas the rest are secondary cases [9]. The incidence of primary UEDVT is about $2-10$ per 100,000 person years $[10,11]$.

Secondary UEDVT is caused by central venous devices, trauma, and cancer. Up to $70 \%$ of UEDVT cases have been correlated with the use of central venous catheters [5] and several studies have reported a cancer prevalence of $22-38 \%$ in UEDVT patients [2, 12-15].
Pulmonary embolism (PE) is a severe side effect following both primary and secondary UEDVT, where rates of up to $20 \%$ have been reported [14-23]. Moreover, a significant proportion, 7-6\% [24] of the patients, might develop the postthrombotic syndrome (PTS). Symptoms of PTS are pain, swelling, edema, varicose veins, and occasional ulcer formation[25].

In order to get a more in-depth understanding of the comorbidity and outcome for patients with primary UEDVT, we conducted a nationwide population-based study in Sweden with a long- term followup.

\section{Methods}

\section{Setting}

We conducted a nationwide, population-based case control study in Sweden using data from the Swedish National Patient Register (NPR) [26]. A tendigit national registration number, a unique identifier assigned to all Swedish residents at birth or immigration, allows record linkages between registries [27].

\section{Description of the Registry}

The cohort was identified in the NPR, which was established by the Swedish National Board of Health and Welfare in 1964. The registry has a practically nationwide coverage for all inpatient visits since 1987. Outpatient visits and ambulatory care have been recorded since 2001. The NPR documents all individual hospital discharges, with each record corresponding to a single hospital episode. Every record contains age, sex, dates of admission and discharge, geographical location of the hospital, clinical ward information, main and contributory discharge codes, and surgical procedures. All diagnoses are coded according to the International Classification of Diseases (ICD-7 until 1968, ICD-8 from 
1969 to 1986 , ICD-9 from 1987 to 1996 , and ICD-10 thereafter).

\section{Definition of Cases}

Data on all patients with ICD codes presented in Table 1 were collected from the NPR for both in- and outpatients during the years 2001-2011. From these data, we identified all patients 18 years of age or older. Patients diagnosed with cancer, orthopedic procedures on the shoulder and upper arm, and devices in the vessel within a period of two years before the index point (the first time that any of the codes presented in Table 1 were registered in the NPR) were excluded in both case and control groups. We assumed that the majority of all important events that could influence the subclavian area were normalized after two years. Therefore, the first index point for inclusion was after 2002. To get a follow-up period of a minimum of three years, only patients with their first thrombotic event reported in the NPR before 2009 were included. Thus, the inclusion period extended from the beginning of 2003 to the end of 2008.

For patients with more than one thrombotic event, the first one was regarded as the index point.

There are specific ICD codes for thromboembolism with various locations, i.e., the lower extremities, the portal vein, etc., but no specific code for UEDVT. Therefore, we considered it important to validate the registry data and 600 patients were randomly selected from a total of 46,349 individuals with thromboembolisms, using the random number generator in IBM SPSS. Their medical records were ordered from the identified hospitals, from which $520(87 \%)$ were handed in. Out of these 520 records, $25(5 \%)$ confirmed the diagnosis of primary UEDVT. The diagnosis was confirmed, according to the medical records, by ultrasound or venography. The review also included documentation of the initial treatment given. These 25 patients were considered to be cases in the study and were compared with the control group.

Table 1. ICD codes for inclusion in study, cases.

\begin{tabular}{|l|l|}
\hline I80.8 & Phlebitis and thrombophlebitis of other sites \\
\hline I80.9 & Phlebitis and thrombophlebitis of unspecified site \\
\hline I82.8 & Embolism and thrombosis of other specified veins \\
\hline I82.9 & Embolism and thrombosis of unspecified vein \\
\hline
\end{tabular}

\section{Definition of Controls}

Patients with acute appendicitis were used as controls. The controls were included from the same source and same period of time as the cases. The ICD-10 codes shown in Table 2 were used. 
Table 2. ICD codes for inclusion, controls.

\begin{tabular}{|l|l|}
\hline K35.0 & Acute appendicitis with generalized peritonitis \\
\hline K35.1 & Acute appendicitis with peritoneal abscess \\
\hline K35.2 & Acute appendicitis with generalized peritonitis \\
\hline K35.3 & Acute appendicitis with localized peritonitis \\
\hline K35.8 & Acute appendicitis, other and unspecified \\
\hline K35.9 & Acute appendicitis, unspecified \\
\hline K36.9 & Other appendicitis \\
\hline K37.9 & Unspecified appendicitis \\
\hline
\end{tabular}

The first time that any of these ICD codes were reported in the NPR was considered to be the index point. Approximately $20 \%$ of the controls had more than one registration referable to appendicitis and, for these, the last registration was classified as the index point. These registrations were adjacent in time, i.e., less than 5 days apart, and were interpreted to be of the same disease episode. For appendicitis, there are specific ICD codes and, therefore, there was no need to review medical records.

\section{Matching Cases-Controls}

The 25 cases were matched, on an individual basis, with 474 controls who were randomly selected from the individuals who were matched according to sex, the same age at the index point as, and same year of, the index point. By this method, the groups were also comparable for the observation period, i.e., the period from the index point to the end of year 2011.

\section{Definition of Comorbidity}

Comorbidity was defined as the number of unique ICD codes (diagnoses) registered in the NPR in each ICD group for cases and controls from the index point to the end of the year 2011. For a better overview, some ICD chapters were merged into ICD groups (study-specific).

The number of healthcare contacts was recorded as the number of admissions to hospital or of outpatient visits.

\section{Ethical Statement}

The study was approved by the Regional Ethical Review Board in Stockholm and it has therefore been conducted in accordance with the ethical standards laid down in the 1964 Declaration of Helsinki and its later amendments. The article is a register study and informed consent is not mandatory according to the Regional Ethical Review Board in Stockholm, Sweden.

\section{Statistics}

The statistical analyses were performed using IBM SPSS Statistics, version 23. Differences in proportions between cases and controls were tested with Fisher's exact test.

Differences in the mean of healthcare contacts were tested with an independent $t$ test. All tests were two-sided and a $\mathrm{P}$ value of $<0.05$ was considered to be statistically significant.

\section{Results}

A total of 25 cases and 474 controls were included in the study. Of these, $48 \%$ were men and $52 \%$ women. The mean age at the index point was 48 years (range, 20-80 yrs; SD, 17.5). The women were slightly older (Figure 1.). 
Medical Research Archives, Vol. 5, Issue 6, June 2017

Primary arm thrombosis - a register study

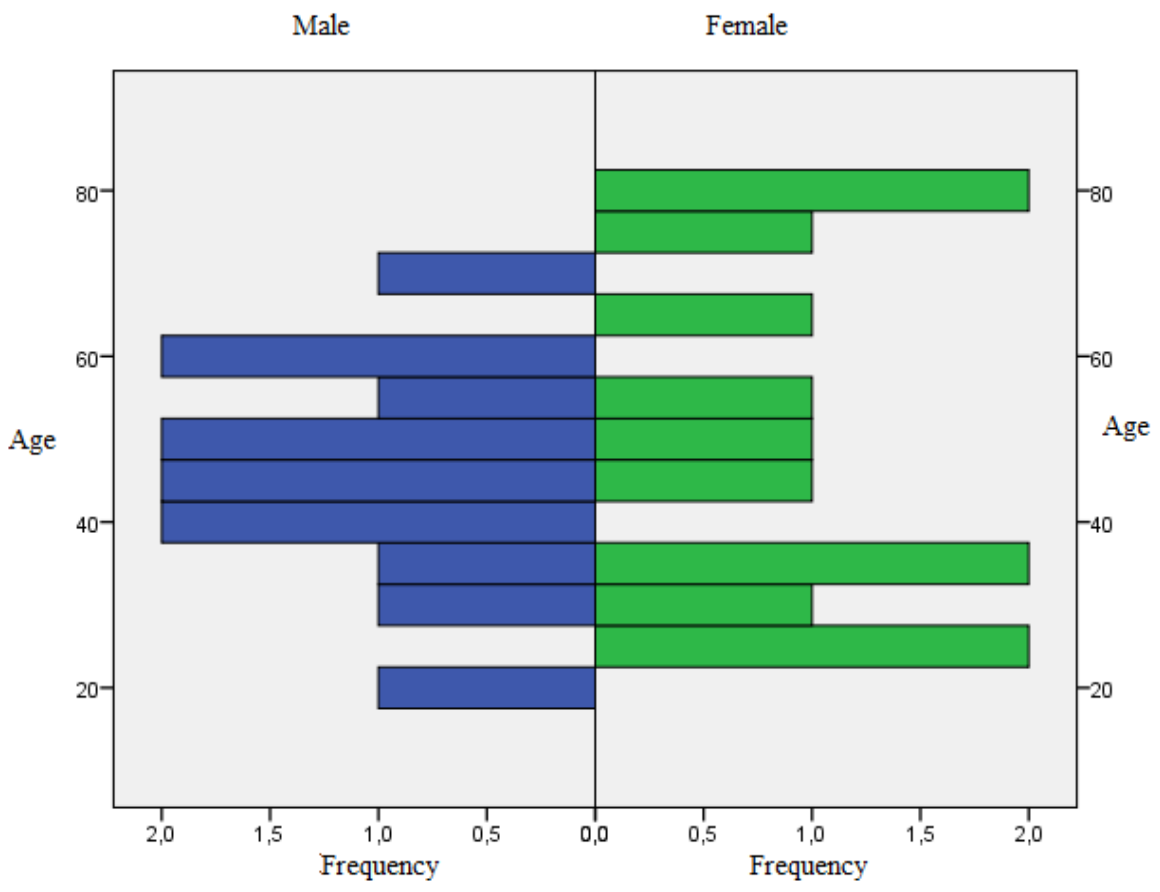

Fig. 1. Age at index point, cases.

The mean follow-up time was 8 years (range, 5.7-9 yrs). All cases were treated with low molecular weight heparin (LMWH) and vitamin $\mathrm{K}$ antagonist (VKA); none underwent initial thrombolysis.

The 25 cases had 561 healthcare contacts (hospital admissions and outpatient visits) during the follow-up period. The cases had a mean of 22 contacts per case (range, 1-97; SD, 21.7), as compared to 9 contacts for each control (range, 1-260; SD, 16.7). The difference was statistically significant, $\mathrm{P}=0.007$

The number of unique ICD codes for cases and controls are presented in Table 3.

On comparing the total number of unique ICD codes, there were no significant differences between cases and controls.

In the diagnoses relating to malignancy, there was no difference between cases and controls.

There was a significant difference between cases and controls for the following
ICD chapters/co-morbidities: Diseases of the blood and blood-forming organs $(\mathrm{P}=0.023)$, Endocrine and metabolic diseases $(\mathrm{P}=$ $0.045)$, Disease of the nervous system $(\mathrm{P}=$ $0.001)$, Diseases of the circulatory system ( $P$ $=0.000)$, Diseases of the skin $(P=0.049)$, and Diseases of the musculoskeletal system $(\mathrm{P}=0.000)$.

Among patients with diagnoses related to the nervous system, $28 \%$ of the cases had symptoms from the brachial plexus or carpal tunnel syndrome, compared with $1.5 \%$ for the controls. The major differences between cases and controls were in diagnoses involving the circulatory system, where $80 \%$ of the cases had such ICD codes while, for controls, the same figure was 16\%. One patient was diagnosed with pulmonary embolism (PE) within one day of the index point.

In chapter 12 , comprising symptoms from the musculoskeletal system, there was an over-representation of ICD codes referring to different diagnoses involving pain and discomfort of the upper extremity. 
Medical Research Archives, Vol. 5, Issue 6, June 2017

Primary arm thrombosis - a register study

For the ICD chapters including diagnoses related to the blood and blood organs, and clear trend for overrepresentation of any endocrine and skin diseases, there was no specific ICD code.

Table 3. Number and percent of unique ICD codes for each ICD group for patients and controls from the index point to the end of year 2011.

\begin{tabular}{|c|c|c|c|c|c|c|c|}
\hline \multirow{2}{*}{$\begin{array}{l}\text { ICD- } \\
\text { Group }\end{array}$} & \multirow[t]{2}{*}{ ICD chapter in ICD 10} & & \multicolumn{2}{|c|}{ Cases } & \multicolumn{2}{|c|}{ Controls } & \multirow[t]{2}{*}{ P value } \\
\hline & & & $\mathbf{n}$ & $\%$ & $\mathbf{n}$ & $\%$ & \\
\hline \multirow[t]{2}{*}{1} & 1. Certain infectious and parasitic diseases & yes & 2 & $8 \%$ & 27 & $6 \%$ & 0.650 \\
\hline & & no & 23 & $92 \%$ & 447 & $94 \%$ & \\
\hline \multirow[t]{2}{*}{2} & 2. Neoplasms & yes & 4 & $16 \%$ & 77 & $16 \%$ & 1.0 \\
\hline & & no & 21 & $84 \%$ & 397 & $84 \%$ & \\
\hline \multirow[t]{2}{*}{3} & 3. Diseases of the blood and blood-forming organs and certain & yes & 3 & $12 \%$ & 10 & $2 \%$ & $0.023^{*}$ \\
\hline & disorders involving the immune mechanism & no & 22 & $88 \%$ & 464 & $98 \%$ & \\
\hline \multirow[t]{2}{*}{4} & 4. Endocrine, nutritional, and metabolic diseases & yes & 5 & $20 \%$ & 36 & $8 \%$ & $0.045^{*}$ \\
\hline & & no & 20 & $80 \%$ & 438 & $92 \%$ & \\
\hline \multirow[t]{2}{*}{5} & 5. Mental and behavioral disorders & yes & 5 & $20 \%$ & 39 & $8 \%$ & 0.059 \\
\hline & & no & 20 & $80 \%$ & 435 & $92 \%$ & \\
\hline \multirow[t]{2}{*}{6} & 6. Diseases of the nervous system & yes & 8 & $32 \%$ & 35 & $7 \%$ & $0.001 *$ \\
\hline & & no & 17 & $68 \%$ & 439 & $93 \%$ & \\
\hline \multirow[t]{2}{*}{7} & 7-8. Diseases of the eye and adnexa and & yes & 5 & $20 \%$ & 86 & $18 \%$ & 0.792 \\
\hline & ear and mastoid process & no & 20 & $80 \%$ & 388 & $82 \%$ & \\
\hline \multirow[t]{2}{*}{8} & 9. Diseases of the circulatory system & yes & 20 & $80 \%$ & 76 & $16 \%$ & $0.000 *$ \\
\hline & & no & 5 & $20 \%$ & 398 & $84 \%$ & \\
\hline \multirow[t]{2}{*}{9} & 10. Diseases of the respiratory system & yes & 3 & $12 \%$ & 35 & $7 \%$ & 0.426 \\
\hline & & no & 22 & $88 \%$ & 439 & $93 \%$ & \\
\hline \multirow[t]{2}{*}{10} & 11. Diseases of the digestive system & yes & 5 & $20 \%$ & 107 & $23 \%$ & 1.000 \\
\hline & & no & 20 & $80 \%$ & 367 & $77 \%$ & \\
\hline \multirow[t]{2}{*}{11} & 12. Diseases of the skin and subcutaneous tissue & yes & 8 & $32 \%$ & 75 & $16 \%$ & $0.049 *$ \\
\hline & & no & 17 & $68 \%$ & 399 & $84 \%$ & \\
\hline \multirow[t]{2}{*}{12} & 13. Diseases of the musculoskeletal system and connective & yes & 15 & $60 \%$ & 113 & $24 \%$ & $0.000 *$ \\
\hline & tissue & no & 10 & $40 \%$ & 361 & $76 \%$ & \\
\hline \multirow[t]{2}{*}{13} & 14. Diseases of the genitourinary system & yes & 5 & $20 \%$ & 83 & $18 \%$ & 0.787 \\
\hline & & no & 20 & $80 \%$ & 391 & $82 \%$ & \\
\hline \multirow[t]{2}{*}{14} & 15. Pregnancy, childbirth, and the puerperium & yes & 1 & $4 \%$ & 25 & $5 \%$ & 1.000 \\
\hline & & no & 24 & $96 \%$ & 449 & $95 \%$ & \\
\hline \multirow[t]{2}{*}{15} & 16. Certain conditions originating in the perinatal period & yes & 0 & $0 \%$ & 0 & $0 \%$ & 1.000 \\
\hline & & no & 25 & $100 \%$ & 474 & $100 \%$ & \\
\hline \multirow[t]{2}{*}{16} & 17. Congenital malformations, deformations, and chromosomal & yes & 0 & $0 \%$ & 4 & $1 \%$ & 1.000 \\
\hline & abnormalities & no & 25 & $100 \%$ & 470 & $99 \%$ & \\
\hline \multirow[t]{8}{*}{17} & 18-21. Symptoms, signs, and abnormal clinical and laboratory & yes & 22 & $88 \%$ & 313 & $66 \%$ & $0.027^{*}$ \\
\hline & findings, not elsewhere classified & no & 3 & $12 \%$ & 161 & $34 \%$ & \\
\hline & $\begin{array}{l}\text { Injury, poisoning and certain other consequences of external } \\
\text { causes }\end{array}$ & & & & & & \\
\hline & External causes of morbidity and mortality & & & & & & \\
\hline & $\begin{array}{l}\text { Factors influencing health status and contact with health } \\
\text { services }\end{array}$ & & & & & & \\
\hline & Codes for special purposes & & & & & & \\
\hline & All ICD codes together & yes & 24 & $96 \%$ & 392 & $83 \%$ & 0.099 \\
\hline & & no & 1 & $4 \%$ & 82 & $17 \%$ & \\
\hline
\end{tabular}




\section{Discussion}

This study is, to our knowledge, the first one in which long-term comorbidity has been described for patients with primary UEDVT. The results show that the comorbidity for malignancies is in line with that of the general population and support our and other authors' opinion that primary UEDVT is a benign disease [28, 29]. However, the cases had a higher number of healthcare contacts.

The relatively high difference between cases and controls in the ICD chapter relating to diseases of the circulatory system is probably explained by the follow-up and monitoring of treatment of the initial thrombotic event.

Kahn et al. [30] and Czihal et al. [31] demonstrated in their studies that PTS has an impact on the quality of life, with upper extremity functional impairment. In the present study, we have not found any evidence of an increased total unique comorbidity, but there was an overrepresentation of diagnoses of symptoms from the musculoskeletal system, brachial plexus, and carpal tunnel syndrome. This can support their findings.

In a previous study by our group [32] with MRI examinations of the thoracic outlet, we found a narrowing of the distance from the clavicle to the first rib (the area where the vessels and nerve pass) also for patients with noneffort-related UEDVT. This raises the question of whether the reported symptoms from the musculoskeletal system of the upper extremity are actually related to the thrombotic event or are due to anatomic conditions of the thoracic outlet. Further examinations with electromyogram (EMG) and electroneurography $(\mathrm{ENoG})$ could be of interest.

In previous studies by our group [28] and Linblad et al. [11] on patients with primary UEDVT, none of the patients had to change occupation after the thrombotic event. This raises the question of the severity of the reduction of the quality of life and its association with physical activity and the patients' expectations pertaining to activity. This issue requires further investigation.

The proper treatment has been widely debated, and some authors recommend prompt thrombolysis [33] [34] due to the importance of patient vessels for reduction of the symptoms. There are, however, no randomized studies on the subject and no convincing proof for the reduction of PTS after thrombolytic therapy has been reported $[35,36]$.

The current guideline from the American College of Chest Physicians (ACCP) suggests that thrombolysis may be beneficial for patients with severe symptoms lasting for less than 14 days, good functional status, and a low bleeding risk [37], but, for the majority of the patients, treatment with LMWH, followed by VKA, is sufficient. The cases in the present study seem to have been treated in concordance with the above guidelines and none of the 25 patients were treated with thrombolysis initially. The rate of PE among patients with primary UEDVT seems to be low, $1-3 \%[11,28]$; in this Study, the rate was one out of 25 patients diagnosed with PE the day after the index point.

The study design is that of a retrospective case-control study comprising of patients with acute appendicitis as controls, selected in the same way as cases and representing morbidity in the general population. A critical characteristic requirement of subject selection in cohort studies is to have both the exposed and unexposed groups selected from the same source population. Subjects who are at risk for developing the outcome should be excluded from the study. Patients with 
appendicitis are perceived to reflect the same exposure pattern as the general population and appendicitis or appendectomy is not related to the causal pathway for the development of primary UEDVT. By choosing patients with acute appendicitis as controls, we minimized the risk of bias and confounding factors.

\section{Limitations of the Study}

The most important limitation is the small number of patients enrolled. Our intention was to have an essentially higher number of included cases. Our hypothesis was that, by selecting the ICD codes, we could achieve conformity between the registry data and the patients' medical records of up to $50-60 \%$, which has been found in previous studies. Two studies on deep venous vein thrombosis (DVT), one from Sweden by Johansson et al. [38] and another from Denmark by Severinsen et al. [39], both with registry data from National Patient Registers, found a frequency of false positive cases of $35-40 \%$. The frequency in the present paper was much higher, $95 \%$. This is because there is no unique code for primary UEDVT in ICD-10 which is used in clinical practice in Sweden. Another finding is that a "DVT-ICD code" is registered when the physician suspects a DVT on clinical grounds, and more specific diagnostic examinations, such as CDU or venography, conducted the following day and the DVT diagnosis can often be written off, but the ICD code remains in the NPR. In addition, both Johansson and Sverinsen had much wider inclusion criteria with all kinds of DVT (i.e., DVT in both arms and legs, primary and secondary, and patients with pulmonary embolism).
The small number of patients enrolled could have been solved with an entirely new and larger random selection of patients and by validating the registry data with their medical records. For methodological reasons, we did not want to add on another random selection to the first one. Another limitation of the study is that there was no way to trace recurrences through the NPR, since a recurrent deep vein thrombosis will receive the same ICD code as the initial event and it is also the same code for the follow-up visits.

\section{Conclusion}

In summary, this study indicates that primary UEDVT is a benign disease. None of the cases developed a malignancy during the observation period (mean, 8 years). There was no difference between patients and controls for the total unique diagnoses reported in the NPR, but there were differences in some ICD chapters that are related to the thrombotic event. Furthermore, the study shows that registry-based data from the NPR must be verified from medical records due to the fact that there is no unique ICD code for primary UEDVT.

\section{Funding and conflict of interest}

This study was funded by Skandinaviska Forskningsstifelsen för Åderbråck och Vensjukdomar (Scandinavian research Foundation for varicose veins and venous diseases). Conflict of Interest: None of the authors declare any conflict of interest. 
Medical Research Archives, Vol. 5, Issue 6, June 2017

Primary arm thrombosis - a register study

\section{References}

1. Joffe, H.V. and S.Z. Goldhaber, Upperextremity deep vein thrombosis. Circulation, 2002. 106(14): 1874-80.

2. Joffe, H.V., et al., Upper-extremity deep vein thrombosis: a prospective registry of 592 patients. Circulation, 2004. 110(12): 1605-11.

3. Thomas, I.H. and B.K. Zierler, An integrative review of outcomes in patients with acute primary upper extremity deep venous thrombosis following no treatment or treatment with anticoagulation, thrombolysis, or surgical algorithms. Vasc Endovascular Surg, 2005. 39(2): 163 74.

4. Hingorani, A., et al., Morbidity and mortality associated with brachial vein thrombosis. Ann Vasc Surg, 2006. 20(3): 297-300.

5. Bernardi, E., R. Pesavento, and P. Prandoni, Upper extremity deep venous thrombosis. Semin Thromb Hemost, 2006. 32(7): 729-36.

6. Kroger, K., et al., Colour Doppler sonographic diagnosis of upper limb venous thromboses. Clin Sci (Lond), 1998. 94(6): 657-61.

7. Lindblad, B., et al., Venous haemodynamics of the upper extremity after subclavian vein thrombosis. Vasa, 1990. 19(3): 218-22.

8. Mustafa, B.O., et al., Sensitivity and specificity of ultrasonography in the diagnosis of upper extremity deep vein thrombosis: a systematic review. Arch Intern Med, 2002. 162(4): 401-4.

9. Bleker, S.M., et al., Clinical course of upper extremity deep vein thrombosis in patients with or without cancer: $a$ systematic review. Thromb Res, 2016. 140 Suppl 1: S81-8.

10. Feugier, P. and J.M. Chevalier, The Paget-Schroetter syndrome. Acta Chir Belg, 2005. 105(3): 256-64.

11. Lindblad, B., L. Tengborn, and D. Bergqvist, Deep vein thrombosis of the axillary-subclavian veins: epidemiologic data, effects of different types of treatment and late sequelae. Eur J Vasc Surg, 1988. 2(3): 161-5.

12. Blom, J.W., et al., Old and new risk factors for upper extremity deep venous thrombosis. J Thromb Haemost, 2005. 3(11): 2471-8.

13. Flinterman, L.E., et al., Current perspective of venous thrombosis in the upper extremity. J Thromb Haemost, 2008. 6(8): 1262-6.

14. Hingorani, A., et al., Risk factors for mortality in patients with upper extremity and internal jugular deep venous thrombosis. J Vasc Surg, 2005. 41(3): 476-8.

15. Munoz, F.J., et al., Clinical outcome of patients with upper-extremity deep vein thrombosis: results from the RIETE Registry. Chest, 2008. 133(1): 143-8.

16. Becker, D.M., J.T. Philbrick, and F.B.t. Walker, Axillary and subclavian venous thrombosis. Prognosis and treatment. Arch Intern Med, 1991. 151(10): 1934-43.

17. Elliott, G., Upper-extremity deep vein thrombosis. Lancet, 1997. 349(9060): 1188-9. 
18. Hingorani, A., et al., Upper extremity deep venous thrombosis and its impact on morbidity and mortality rates in a hospital-based population. J Vasc Surg, 1997. 26(5): 853-60.

19. Horattas, M.C., et al., Changing concepts of deep venous thrombosis of the upper extremity--report of a series and review of the literature. Surgery, 1988. 104(3): 561-7.

20. Kooij, J.D., et al., Pulmonary embolism in deep venous thrombosis of the upper extremity: more often in catheterrelated thrombosis. Neth J Med, 1997. 50(6): 238-42.

21. Monreal, M., et al., Pulmonary embolism in patients with upper extremity DVT associated to venous central lines--a prospective study. Thromb Haemost, 1994. 72(4): 548-50.

22. Prandoni, P., et al., Upper-extremity deep vein thrombosis. Risk factors, diagnosis, and complications. Arch Intern Med, 1997. 157(1): 57-62.

23. Levy, M.M., F. Albuquerque, and J.D. Pfeifer, Low incidence of pulmonary embolism associated with upperextremity deep venous thrombosis. Ann Vasc Surg, 2012. 26(7): 964-72.

24. Elman, E.E. and S.R. Kahn, The postthrombotic syndrome after upper extremity deep venous thrombosis in adults: a systematic review. Thromb Res, 2006. 117(6): 609-14.

25. Kahn, S.R., A. Hirsch, and I. Shrier, Effect of postthrombotic syndrome on health-related quality of life after deep venous thrombosis. Arch Intern Med, 2002. 162(10): 1144-8.
26. National Patient Register in Sweden.

27. Ludvigsson, J.F., et al., Registers of the Swedish total population and their use in medical research. Eur J Epidemiol, 2016. 31(2): 125-36.

28. Arnhjort, T., et al., Primary deep vein thrombosis in the upper limb: A retrospective study with emphasis on pathogenesis and late sequelae. Eur $\mathrm{J}$ Intern Med, 2007. 18(4): 304-8.

29. Spencer, F.A., et al., Upper extremity deep vein thrombosis: a communitybased perspective. Am J Med, 2007. 120(8): 678-84.

30. Kahn, S.R., et al., Post-thrombotic syndrome, functional disability and quality of life after upper extremity deep venous thrombosis in adults. Thromb Haemost, 2005. 93(3): 499502.

31. Czihal, M., et al., Impact of the postthrombotic syndrome on quality of life after primary upper extremity deep venous thrombosis. Vasa, 2012. 41(3): 200-4.

32. Arnhjort, T., et al., The importance of the costoclavicular space in upper limb primary deep vein thrombosis, a study with magnetic resonance imaging (MRI) technique enhanced by a blood pool agent. European Journal of Internal Medicine, 2014. 25(6): 545549.

33. van den Houten, M.M., et al., Treatment of upper-extremity outflow thrombosis. Phlebology, 2016. 31(1 Suppl): 28-33.

34. Thompson, R.W., Comprehensive management of subclavian vein effort 
Medical Research Archives, Vol. 5, Issue 6, June 2017

Primary arm thrombosis - a register study

thrombosis. Semin Intervent Radiol, 2012. 29(1): 44-51.

35. Sabeti, S., et al., Treatment of subclavian-axillary vein thrombosis: long-term outcome of anticoagulation versus systemic thrombolysis. Thromb Res, 2002. 108(5-6): 279-85.

36. Bosma, J., et al., Primary subclavian vein thrombosis and its long-term effect on quality of life. Vascular, 2011. 19(6): 327-32.

37. Kearon, C., et al., Antithrombotic therapy for VTE disease: Antithrombotic Therapy and
Prevention of Thrombosis, 9th ed: American College of Chest Physicians Evidence-Based Clinical Practice Guidelines. Chest, 2012. 141(2 Suppl): 419S-94S.

38. Johansson, M., L. Johansson, and M. Lind, Incidence of venous thromboembolism in northern Sweden (VEINS): a population-based study. Thromb J, 2014. 12(1): 6.

39. Severinsen, M.T., et al., Venous thromboembolism discharge diagnoses in the Danish National Patient Registry should be used with caution. J Clin Epidemiol, 2010. 63(2): 223-8. 